\title{
Radiative Transfer Simulations of Infrared Dark Clouds
}

\author{
Yaroslav Pavlyuchenkov ${ }^{1}$, Dmitry Wiebe ${ }^{1}$, Anna Fateeva $^{1}$, \& Tatiana \\ Vasyunina $^{2}$ \\ ${ }^{1}$ Institute of Astronomy, Russian Academy of Sciences, \\ Pyatniskaya str. 48, 119017 Moscow, Russia \\ email: pavyar@inasan.ru \\ ${ }^{2}$ Max Planck Institute for Astronomy, \\ Koenigstuhl 17, D-69117 Heidelberg, Germany \\ email: vasyunina@mpia.de
}

\begin{abstract}
The determination of prestellar core structure is often based on observations of (sub)millimeter dust continuum. However, recently the Spitzer Space Telescope provided us with IR images of many objects not only in emission but also in absorption. We developed a technique to reconstruct the density and temperature distributions of protostellar objects based on radiation transfer (RT) simulations both in $\mathrm{mm}$ and IR wavelengths. Best-fit model parameters are obtained with the genetic algorithm. We apply the method to two cores of Infrared Dark Clouds and show that their observations are better reproduced by a model with an embedded heating source despite the lack of $70 \mu \mathrm{m}$ emission in one of these cores. Thus, the starless nature of massive cores can only be established with the careful case-by-case RT modeling.
\end{abstract}

Keywords. radiative transfer; ISM: clouds, dust, extinction; methods: data analysis, numerical;

\section{Objectives}

Parameters of prestellar and protostellar molecular cores are usually derived from millimeter and sub-millimeter observations of their thermal dust emission. However, the interpretation of these observations is ambiguous as the same spectrum can be produced by different density and temperature distributions. In some cases, millimeter continuum data are supplemented by molecular line observations to derive the temperature. But the interpretation of molecular line observations is also not trivial because of the non-LTE conditions of line formation and complex chemical structure of the cores. The Spitzer Space Telescope provided us with infrared images of many dense cores, seen not only in emission but also in absorption. These data can be used to derive the core density and temperature distributions by fitting absorption and emission simultaneously.

We present a framework to reconstruct the density and temperature distributions of protostellar objects based on detailed RT simulations and on the comparison of model intensity distributions with observations both in emission and absorption at mm and IR wavelengths. We apply the method to two Infrared Dark Cloud cores IRDC$320.27+029$ (subcore P2) and IRDC-321.73+005 (subcore P2) which are believed to represent the initial stage for the massive star formation (Fig. 1). For brevity, they are further referred to as IRDC 320 and IRDC 321. 

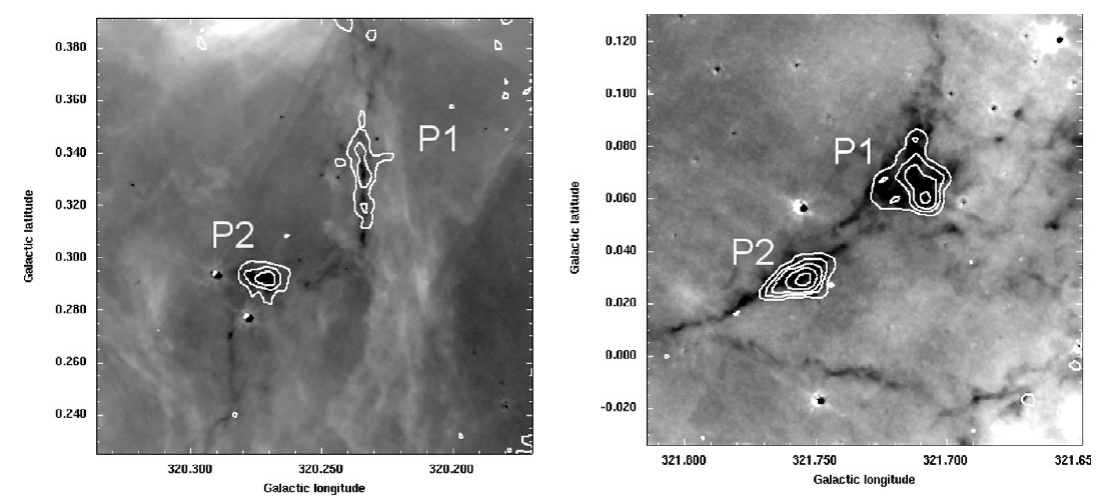

Figure 1. Composite images of dark cores IRDC-320.27+029 and IRDC-321.73+005. The grey map is for $8 \mu \mathrm{m}$ emission provided by the Spitzer Space Telescope while contours show $1.2 \mathrm{~mm}$ emission from the SEST Telescope.
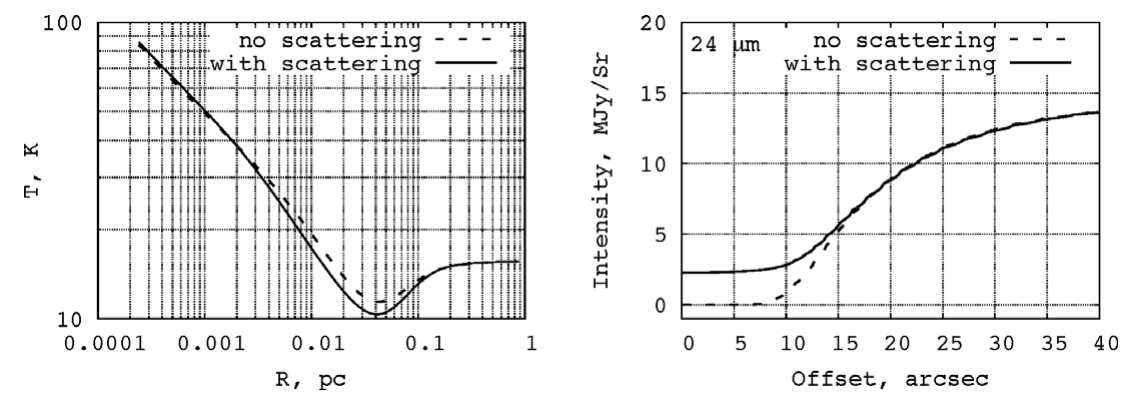

Figure 2. Temperature and intensity distributions for a representative model of a protostellar core. The central heating source is responsible for the inner temperature gradient while the external radiation field heats the envelope. The solid and dashed lines correspond to the models with and without scattering, correspondingly.

\section{Model of a protostellar core}

We assume that a core is spherically symmetric and its density distribution can be represented by following law, see Tafalla et al. (2002):

$$
n\left(\mathrm{H}_{2}\right)=\frac{n_{0}}{1+\left(r / r_{0}\right)^{\beta}},
$$

where $n_{0}$ is the central density, $r_{0}$ is the radius of inner plateau, $\beta$ describes the density decrease in the envelope. We also set the inner core radius of $50 \mathrm{AU}$ and the outer radius of $1 \mathrm{pc}$. To take into account the possible spread in evolutionary stages, e.g. the presence of a (proto)star, we assume that there is a blackbody source in the core center with the temperature of $T_{*}$ and radius of $5 R_{\odot}$. The core is illuminated by the diffuse interstellar field with the color temperature $10000 \mathrm{~K}$ and dilution $D_{\mathrm{bg}}$. This field controls the temperature in the envelope. We also postulate the isotropic infrared background emission for those wavelengths where the comparison with observations has to be made. The intensity of infrared background field is equal to the observed intensity at the edge of the core. Note that it is much stronger than the diluted interstellar field.

Temperature distribution and emergent intensity distribution are found as a result of RT simulation with the NATA(LY) code by Pavlyuchenkov et al. (2010) for the given set of parameters. Dust emission, absorption and isotropic scattering are taken into account. The accelerated $\Lambda$-iteration method is used where the mean intensity is calculated using 

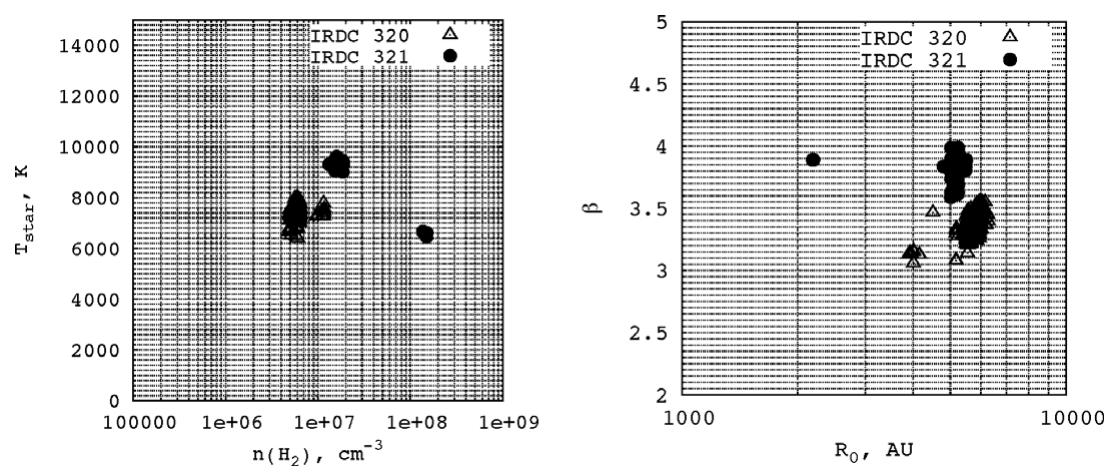

Figure 3. Localization of IRDC 320 and IRDC 321 model parameters with $\chi^{2}<11$ found by PIKAIA code. In the case of IRDC 320 the parameters are well localized while for IRDC 321 there are two groups of parameters with nearly the same $\chi^{2}$.

the set of selected rays. The frequency dependent dust opacities are calculated from the Mie theory. Temperature and intensity distributions for a representative core model are shown in Fig. 2. Note that scattering does not significantly affect the thermal structure but can strongly affect the emergent intensity distribution.

\section{Search for best-fit parameters}

For each set of free parameters we perform RT simulations and calculate the model intensity distributions for $1.2 \mathrm{~mm}, 70 \mu \mathrm{m}, 24 \mu \mathrm{m}$, and $8 \mu \mathrm{m}$. Then we calculate the $\chi^{2}$ criterion which characterizes the agreement between the model and observed intensity distributions. Millimeter data are taken from Vasyunina et al. (2009). Infrared data are downloaded from NASA/IPAC Infrared Science Archive (http://irsa.ipac.caltech.edu/).

The search for best-fit parameters i.e. the minimization of $\chi^{2}$ is performed using the genetic code PIKAIA, see Charbonneau (1995). This algorithm allows not only to identify the global minimum of $\chi^{2}$ in the multi-dimensional parameter space but also to study the degeneracy of the solution. The localization of the optimal parameters (with $\chi^{2}<11$ ) for IRDC 320 and IRDC 321 in two parameter subspaces is shown in Fig. 3. In Table 1 the best-fit, derived and fixed parameters for the studied cores are shown.

\section{Main Results}

In general, we achieved a good agreement between synthetic and observed intensity distributions both for IRDC 320 and IRDC 321 (Fig. 4). In the case of IRDC 320 we are able to fit $1.2 \mathrm{~mm}$ emission profile and to reproduce the flat intensity distribution at 70 $\mu \mathrm{m}$ and absorption profiles at $24 \mu \mathrm{m}$ and $8 \mu \mathrm{m}$. In the model of IRDC 321 the emission appears not only at $1.2 \mathrm{~mm}$ but also at $70 \mu \mathrm{m}$ in agreement with observations. That is a result of higher central density and higher temperature of the inner heating source.

In both cases our results are consistent with the presence of internal heating sources (proto-stars) in cores' interiors. While for IRDC 321 this assertion seems to be robust as it is seen in emission at $70 \mu \mathrm{m}$, for the particular case of IRDC 320 it should only be considered as tentative. In particular, we did not try to distinguish between the foreground and background radiation. Second, available molecular data do not show any typical hot core tracers in this object. So, this case deserves further study, in particular, with chemical models. The important conclusion of this study is that $70 \mu \mathrm{m}$ band seems to be most promising to discriminate between starless and protostellar massive cores. 
Table 1. Best-fit, derived and fixed parameters for template cores

\begin{tabular}{|c|c|c|c|}
\hline Parameter & Name & IRDC 320 & IRDC 321 \\
\hline \multicolumn{4}{|c|}{ Varied parameters } \\
\hline $\mathrm{H}_{2}$ central density, $\mathrm{cm}^{-3}$ & $n_{0}$ & $1.1 \times 10^{7}$ & $1.8 \times 10^{7}$ \\
\hline Radius of plateau, $\mathrm{AU}$ & $r_{0}$ & $4 \times 10^{3}$ & $5 \times 10^{3}$ \\
\hline Index for envelope & $\beta$ & 3.1 & 3.8 \\
\hline Star temperature, $\mathrm{K}$ & $T_{*}$ & 7300 & 9300 \\
\hline Dilution of background field & $D_{\text {bg }}$ & $1.3 \times 10^{-13}$ & $6.7 \times 10^{-14}$ \\
\hline \multicolumn{4}{|c|}{ Derived parameters } \\
\hline Cloud mass, $M_{\odot}$ & $M$ & 170 & 230 \\
\hline $\mathrm{H}_{2}$ surface density, $\mathrm{cm}^{-2}$ & $N$ & $8.1 \times 10^{23}$ & $1.6 \times 10^{24}$ \\
\hline Star luminosity, $L_{\odot}$ & $L$ & 60 & 160 \\
\hline \multicolumn{4}{|c|}{ Fixed parameters } \\
\hline Radius of inner hole & $R_{\text {in }}$ & & \\
\hline Cloud radius & $R_{\text {out }}$ & & pc \\
\hline Star radius & $R_{*}$ & & $R_{\odot}$ \\
\hline Temperature of background field & $T_{\mathrm{bg}}$ & & $\mathrm{K}$ \\
\hline
\end{tabular}
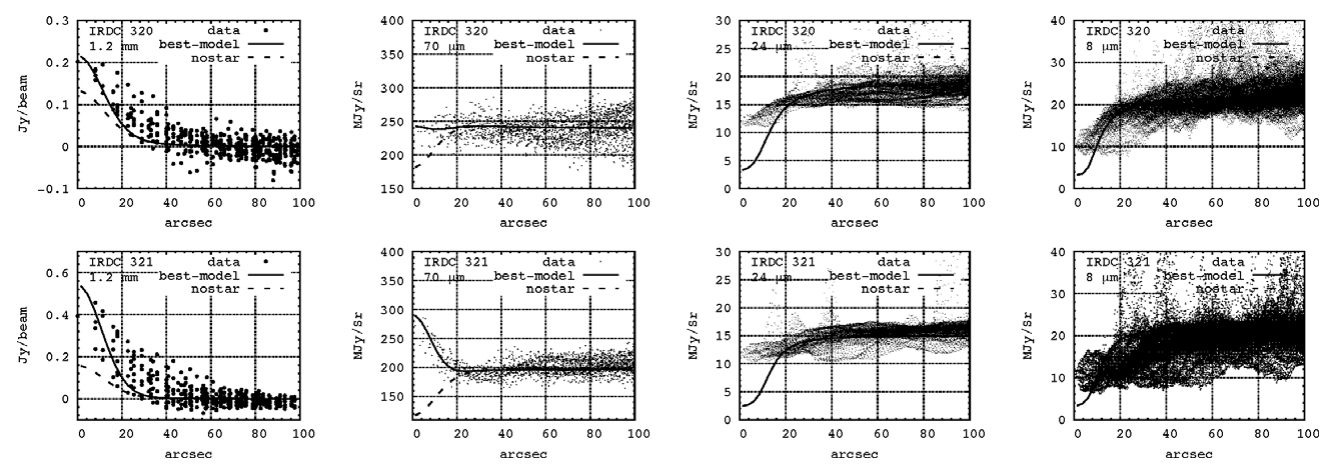

Figure 4. Observed (dots) and modeled (lines) intensity distributions at selected wavelengths for IRDC 320 (top row) and IRDC 321 (bottom row). The model distributions correspond to the model with lowest $\chi^{2}$. With dashed lines, we show results for the same core model, but without the central heating source. It is noteworthy that not only $70 \mu \mathrm{m}$ but also millimeter emission is sensitive to the presence of the heating source.

However, observations in this band should not be interpreted without careful modeling to avoid simplified conclusions. In particular, the mere absence of $70 \mu \mathrm{m}$ emission is not a solid evidence that a core is starless.

This study has been supported by RF President Grant MK-4713.2009.2 and by RFBR grant 10-02-00612.

\section{References}

Charbonneau, P.; 1995, Astroph. J. Suppl., 101, 309

Pavlyuchenkov, Ya.; Wiebe, D.; Fateeva, A.; Vasyunina, T.; 2010, Astron. Reports, in press Tafalla, M., Myers, P. C., Caselli, P., Walmsley, C. M., \& Comito, C.; 2002, ApJ, 569, 815

Vasyunina, T.; Linz, H.; Henning, T. et al.; 2009, A\&A, 499, 149 